\title{
Primer registro de Ulex europaeus (Fabaceae, Faboideae) en México
}

\author{
First record of Ulex europaeus (Fabaceae, \\ Faboideae) in Mexico
}

\section{Acta Botanica Mexicana}

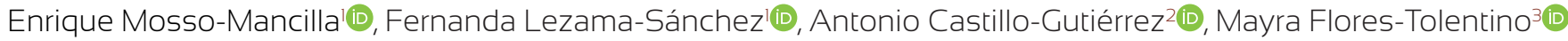

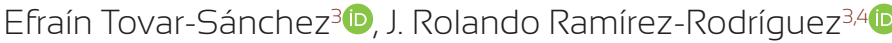

\section{Resumen:}

Antecedentes y Objetivos: La especie Ulex europaeus (Fabaceae, Faboideae) ha sido catalogada como una de las 100 especies invasoras más dañinas a nivel mundial. Es originaria del oeste de Europa y noroeste de África y se ha naturalizado en toda Europa y el Medio Oriente. Su impacto ambiental y económico en América está documentado en Canadá, Chile, Colombia y los Estados Unidos de América. Se encuentra en diferentes altitudes y tipos de vegetación. Con este estudio se busca contribuir al conocimiento de la distribución de Ulex europaeus en el territorio mexicano, como una base para acciones inmediatas respecto a su control y erradicación.

Métodos: Se colectó material botánico en el bosque templado de la comunidad de Tres Marías, municipio Huitzilac, en el estado de Morelos, México. Para la determinación taxonómica se utilizaron claves dicotómicas y descripciones botánicas. Se realizó una revisión exhaustiva de literatura especializada, se consultaron imágenes, se obtuvieron datos de herbarios virtuales del mundo y se visitaron, para su consulta, los herbarios MEXU y UAMIZ. Los recorridos de campo en el estado de Morelos se apoyaron con proyecciones de ArcGIS. El material colectado está depositado en la colección del herbario HUMO de la Universidad Autónoma del Estado de Morelos.

Resultados clave: Los autores registran por primera vez mediante colectas botánicas la presencia de U. europaeus en México. Apoyándose en recorridos en campo y la revisión de varias fuentes de información, hasta el momento solo se ha encontrado una población de 63 individuos de esta especie. La fenología de la especie coincide con lo reportado para otras latitudes.

Conclusiones: Este registro amplía el rango de distribución de $U$. europaeus. La presencia de esta especie se suma a los factores reales y potenciales que están afectando a la biodiversidad de nuestro país. Es necesario establecer estrategias, acciones de control y eventual erradicación de esta especie invasora.

Palabras clave: ArcGIS, biodiversidad, bosque templado, especies invasoras, Morelos.

\section{Abstract:}

Background and Aims: The species Ulex europaeus (Fabaceae, Faboideae) has been listed as one of the 100 most harmful invasive species worldwide. It is native to western Europe and northwestern Africa, and has become naturalized throughout Europe and the Middle East. Its environmental and economic impact in America is documented in Canada, Chile, Colombia and the United States of America. It is found at different altitudes and in vegetation types. This study aims to contribute to the knowledge of the distribution of Ulex europaeus in the Mexican territory, as a basis for immediate actions regarding its control and eradication.

Methods: Botanical material was collected in the temperate forest of the community of Tres Marías, municipality Huitzilac, in the state of Morelos, Mexico. Dichotomous keys and botanical descriptions were used for taxonomic determination. An exhaustive review of specialized literature was carried out, images were consulted, and data were obtained from virtual herbaria around the world, and the herbaria MEXU and UAMIZ were visited for consultation. The field work in the state of Morelos was supported by ArcGIS projections. The collected material is deposited in the collection of the herbarium HUMO of the Autonomous University of the State of Morelos.

Key results: The authors register for the first time, by means of botanical collections, the presence of $U$. europaeus in Mexico. Supported by field trips and the review of various sources of information, so far only a population of 63 individuals of this species has been found. The phenology of the species coincides with that reported for other latitudes

Conclusions: This record broadens the distribution range of $U$. europaeus. The presence of this species adds to the real and potential factors that are affecting the biodiversity of our country. It is necessary to establish strategies, control actions and eventual eradication of this invasive species.

Key words: ArcGIS, biodiversity, invasive species, Morelos, temperate forest.

${ }^{1}$ Universidad Autónoma del Estado de Morelos, Facultad de Ciencias Biológicas (FCB), Av. Universidad 1001, Col. Chamilpa, 62209 Cuernavaca, Morelos, México. ${ }^{2}$ Universidad Autónoma del Estado de Morelos, Es cuela de Estudios Superiores de Xalostoc (EESuX), Interior del Parque Industrial Cuautla sin número, 62715 Ayala, Morelos, México

${ }^{3}$ Universidad Autónoma del Estado de Morelos, Centro de Investigación en Biodiversidad y Conservación (ClByC), Avenida Universidad 1001, Col. Chamilpa, 62209 Cuernavaca, Morelos, México.

${ }^{4}$ Autor para la correspondencia: rolando@uaem.mx
Recibido: 20 de octubre de 2021

Revisado: 3 de noviembre de 2021.

Aceptado por Marie-Stéphanie-Samain: 1 de diciembre de 2021.

Publicado Primero en línea: 19 de enero de 2022.

Publicado: Acta Botanica Mexicana 129(2022).

Este es un artículo de acceso abierto bajo la licencia Creative Commons 4.0 Atribución-No Comercial (CC BY-NC 4.0 Internacional).
Citar como: Mosso-Mancilla, E., F. Lezama-Sánchez, A. Castillo-Gutiérrez, M. Flores-Tolentino, E. Tovar-Sánchez y J. R. Ramírez-Rodríguez. 2022. Primer registro de Ulex europaeus (Fabaceae, Faboideae) en México. Acta Botanica Mexicana 129: e1987. DOI: https://doi. org/10.21829/abm129.2022.1987

e-ISSN: 2448-7589 


\section{Introducción}

La especie Ulex europaeus L. (Fabaceae, Faboideae) es originaria del oeste de Europa y noroeste de África (Clements et al., 2001; Díaz-Espinoza, 2009) y se ha naturalizado en toda Europa y el Medio Oriente. Es tolerante a altitudes con condiciones templadas, pero no a altitudes con temperaturas frías (Cubas et al., 2005; Hill et al., 2008). Se ha establecido en regiones montañosas de Hawái y Sri Lanka (Lowe et al., 2000) y en América se ha registrado en Brasil, Canadá, Chile, Colombia, Costa Rica, Ecuador, Estados Unidos de América, Panamá y Perú (Broadfield y McHenry, 2019). Ulex europaeus es un combustible muy eficiente debido a que acumula una elevada cantidad de ramas secas, lo que le da un carácter pirófilo (Schroeder, 1979, citado por Springer, 1985; Baeza et al., 2002; Díaz-Espinoza, 2009; Madrigal et al., 2012). Las implicaciones de la presencia de $U$. europaeus han sido documentadas de la siguiente manera: como maleza de importancia silvo-agropecuaria (Clements et al., 2001), por su uso de controles biológicos (Beckdorf, 1985; Sandrey, 1985), por su afectación del balance entre el suelo y el agua (Soto y Diaz-Fierros, 1997), por su impacto económico (Leung et al., 2002), y por su impacto en la salud humana (Johnston et al., 2012). En estudios de restauración ecológica (Camelo-Salamanca, 2015) destaca por su papel en la fragmentación del paisaje (Altamirano et al., 2016; Araos et al., 2020) y como proveedor de polen (Bowman et al., 2008). A U. europaeus se le ha dado un uso medicinal (Suguru-Yonezawa et al., 1987; González-Hernández et al., 2004), alimenticio (Wolf, 1988), ornamental (Ramírez-Morán et al., 2016), de combustible (Niño et al., 2018), como cerca viva (Osorio y Cerda, 1984), como estabilizador de suelos (Maxwell, 1995) y como sustrato para horticultura, floricultura y paisajismo (Gómez et al., 2009; López y López, 2012).

En su fenología, $U$. europaeus muestra una alta plasticidad, pues florece prácticamente en cualquier época del año y sus flores producen abundante polen, siendo este el principal recurso ofrecido a sus polinizadores, que son abejas y abejorros (Hill et al., 1991; Tarayre et al., 2007). Cuando inmaduros, sus frutos son vainas suaves y de color verde, al madurar son duros y de color marrón; presentan dehiscencia explosiva (Norambuena y Piper, 2000). Sus semillas duras pueden permanecer inactivas durante más de
20 años (Hill et al., 2001). Es una especie capaz de autofecundarse; sin embargo, no se ha registrado una depresión por endogamia (Herrera, 1999). Hendry y Day (2005) establecen que, de manera general, la capacidad reproductiva expresada en las poblaciones desde estadios juveniles y en cualquier época del año, aunado a los tiempos tan largos de dormancia, permiten una superposición de generaciones que podría estar influyendo en el mantenimiento del polimorfismo genético de la especie; sobre todo si hay una baja presión selectiva (Ellner y Sasaki, 1996). La base del número de cromosomas de $U$. europaeus es 48 y generalmente es hexaploide $(2 n=96)$, pero también existen individuos diploides y tetraploides (Fernández-Prieto et al., 1993; Misset y Gourret, 1996). Esta variabilidad genética establece las bases de la adecuación de las especies, ante los agentes promotores de la evolución como son la mutación, la deriva genética, la migración y la selección natural (Haldane y Jayakar, 1963; Piñero et al., 2008).

Las características genéticas y fenológicas, los antecedentes de registro en diferentes sistemas ambientales, su referencia como una especie invasora de alto impacto negativo en los ecosistemas en los que se establece, el registro en América tanto al norte como al sur de México y la actual corroboración de la presencia de esta especie en el territorio nacional, requieren acciones transdisciplinarias, para evitar, una afectación adicional a la biodiversidad mexicana, generada ahora por Ulex europaeus.

Tres fuentes mencionan la presencia de esta especie en México: 1) la base de datos del Instituto de Silvicultura de las Islas del Pacífico (Philip y Thomas, 2018), 2) la página de Tropicos (Tropicos, 2020) y 3) el trabajo de Atlan y Udo (2019), quienes realizaron un análisis de las publicaciones que aparecen en internet sobre $U$. europaeus y comentan que en México esta especie es considerada una plaga agrícola invasora, con impacto económico y uso medicinal. Sin embargo, Sánchez-Blanco et al. (2012), en su estudio sobre especies de leguminosas con potencial invasor y que han sido introducidas en México, no mencionan la presencia de esta especie en el territorio mexicano. Asimismo, tampoco se encontró información relacionada con localidad, altitud, tipo de vegetación o georreferencia que permitiera constatar su presencia en México en la información revisada en las bases de datos disponibles en las siguientes coleccio- 
nes botánicas: Muséum National d'Histoire Naturelle $(\mathrm{P})(\mathrm{P}$, 2020), New York Botanical Garden (NY) (NY, 2020), Royal Botanic Garden (K) (K, 2021), Missouri Botanical Garden Herbarium (MO) (MO, 2020), Herbario del Komarov Botanical Insititute (LE) (LE, 2021), United States National Herbarium (US) (US, 2020), Herbario Nacional de México (MEXU) (MEXU, 2020), Herbario de la Escuela Nacional de Ciencias Biológicas (ENCB) (ENCB, 2020), y la Base de Datos Global de Especies Invasoras (IUCNGISD, 2020) y Naturalista (Naturalista, 2020).

El género Ulex L. está formado por un complejo de 34 taxones, con 30 especies aceptadas y cuatro híbridos (MNHN, 2021). Sin embargo, la única especie de este género reconocida en América es U. europaeus (LOWO, 2020). Ante esta situación y considerando los antecedentes del impacto negativo que genera esta especie, se fortaleció el interés por realizar este estudio y determinar si lo colectado por los autores en México en la comunidad de Tres Marías en el Municipio Huitzilac, estado de Morelos, es U. europaeus. Además, se consideró importante caracterizar de manera básica las condiciones ambientales de la localidad en donde esta especie se ha registrado, y generar una alerta acerca de las implicaciones negativas de esta especie registradas en otras latitudes; implicaciones que, en caso de ignorar su presencia, podrán replicarse en el territorio nacional.

\section{Materiales y Métodos}

Atendiendo los protocolos para colectas botánicas propuestos por Lot y Chiang (1986) y Bridson y Forman (1998), se colectó material botánico mensualmente durante un año (2019), en la Comunidad de Tres Marías, municipio Huitzilac en el estado de Morelos, México. En la determinación taxonómica se utilizaron claves y descripciones botánicas (Cubas, 1984). Se revisaron presencialmente los herbarios de la Universidad Autónoma Metropolitana-Unidad Iztapalapa UAMIZ, de la Escuela Nacional de Ciencias Biológicas del Instituto Politécnico Nacional ENCB, de la Universidad Nacional Autónoma de México MEXU y virtualmente los herbarios LE del Komarov Botanical Insititute, P del Muséum National d'Histoire Naturelle, NY del New York Botanical Garden, K del Royal Botanic Garden, MO del Missouri Botanical Garden, US del Smithsonian Institution, MEXU de la Universidad Nacional Autónoma de México,
ENCB de la Escuela Nacional de Ciencias Biológicas (ENCB) (ENCB, 2020) (Acrónimos según Thiers, 2021); así como todas las referencias que potencialmente pudieran vincular a $U$. europaeus con México. Con los datos obtenidos por los autores en las colectas botánicas de este estudio y para determinar el potencial de distribución geográfica o ubicación de esta especie en el estado de Morelos, se utilizaron dos capas de sets de datos geográficos: altitud (Cruz-Cárdenas et al., 2014) y biomas de México (Villaseñor y Ortiz, 2014), los cuales fueron procesados en el programa ArcGIS v. 10.2 (ESRI, 2013), donde se extrajeron los valores de cada variable (Cuadro 1). La identificación de sitios en el estado de Morelos, con características similares a las del sitio de colecta del material botánico, permitió trazar la ruta de exploración en la búsqueda de esta especie. El clima de la localidad de la colecta presenta una temperatura promedio de $11.2^{\circ} \mathrm{C}$; el promedio de las temperaturas máximas es de $17.5^{\circ} \mathrm{C}$, y el promedio de las temperaturas mínimas es de $4.8^{\circ} \mathrm{C}$; la precipitación media anual es de $1.517 \mathrm{~mm}$ (SMN, 2019). Dos ejemplares del material colectado se procesaron para su incorporación al acervo botánico del herbario

Cuadro 1: Variables de cada uno de los 15 sitios con las mismas características de vegetación y altitud que el nuevo registro para Ulex europaeus L. obtenidos en el estado de Morelos, México, con el programa ArcGIS v. 10.2 (ESRI, 2013). BTEM: Bosque templado, BHM: Bosque húmedo de montaña.

\begin{tabular}{ccccc}
\hline Sitio & Latitud & Longitud & Bioma & Altitud $(\mathbf{m})$ \\
\hline 1 & $19^{\circ} 3^{\prime} 21.439^{\prime \prime}$ & $-98^{\circ} 56^{\prime} 44.969^{\prime \prime}$ & BTEM & 2663 \\
2 & $19^{\circ} 2^{\prime} 41.441^{\prime \prime}$ & $-99^{\circ} 16^{\prime} 9.923^{\prime \prime}$ & BTEM & 2725 \\
3 & $19^{\circ} 2^{\prime} 41.441^{\prime \prime}$ & $-99^{\circ} 9^{\prime} 54.938^{\prime \prime}$ & BTEM & 2748 \\
4 & $19^{\circ} 2^{\prime} 11.442^{\prime \prime}$ & $-99^{\circ} 14^{\prime} 24.927^{\prime \prime}$ & BTEM & 2709 \\
5 & $19^{\circ} 2^{\prime} 11.442^{\prime \prime}$ & $-99^{\circ} 11^{\prime} 9.935^{\prime \prime}$ & BTEM & 2707 \\
6 & $19^{\circ} 2^{\prime} 11.442^{\prime \prime}$ & $-99^{\circ} 8^{\prime} 39.941^{\prime \prime}$ & BTEM & 2710 \\
7 & $19^{\circ} 2^{\prime} 11.442^{\prime \prime}$ & $-99^{\circ} 0^{\prime} 24.96^{\prime \prime}$ & BTEM & 2665 \\
8 & $19^{\circ} 1^{\prime} 41.443^{\prime \prime}$ & $-99^{\circ} 16^{\prime} 54.921^{\prime \prime}$ & BHM & 2741 \\
9 & $19^{\circ} 1^{\prime} 41.443^{\prime \prime}$ & $-99^{\circ} 6^{\prime} 24.946^{\prime \prime}$ & BTEM & 2700 \\
10 & $19^{\circ} 1^{\prime} 41.443^{\prime \prime}$ & $-99^{\circ} 1^{\prime} 54.957^{\prime \prime}$ & BHM & 2700 \\
11 & $19^{\circ} 1^{\prime} 11.444^{\prime \prime}$ & $-99^{\circ} 18^{\prime} 24.917^{\prime \prime}$ & BHM & 2700 \\
12 & $19^{\circ} 1^{\prime} 11.444^{\prime \prime}$ & $-99^{\circ} 17^{\prime} 24.92^{\prime \prime}$ & BHM & 2714 \\
13 & $18^{\circ} 56^{\prime} 41.455^{\prime \prime}$ & $-98^{\circ} 42^{\prime} 25.004^{\prime \prime}$ & BTEM & 2713 \\
14 & $18^{\circ} 55^{\prime} 41.457^{\prime \prime}$ & $-98^{\circ} 41^{\prime} 55.005^{\prime \prime}$ & BTEM & 2653 \\
15 & $18^{\circ} 55^{\prime} 11.459^{\prime \prime}$ & $-98^{\circ} 42^{\prime} 25.004^{\prime \prime}$ & BTEM & 2670 \\
\hline
\end{tabular}


HUMO (Universidad Autónoma del Estado de Morelos). El resto de los duplicados se han preparado para enviar como intercambio a otros herbarios.

\section{Resultados}

El registro y colecta de material botánico determinado como U. europaeus se realizó en México, estado de Morelos, municipio Huitzilac, específicamente en la comunidad de Tres Marías, cuyas coordenadas de colecta son $19^{\circ} 2^{\prime} 11.734^{\prime \prime} \mathrm{N}$, $99^{\circ} 13^{\prime} 47.475^{\prime \prime O}$, donde la altitud es de $2700 \mathrm{~m}$ en una vegetación templada (alterada) de bosque de Pinus L. con algunos elementos de Quercus L. El material recolectado corresponde a las referencias de colecta de Rolando Ramírez-Rodríguez 5601 y Enrique Mosso-Mancilla s.n., a los que respectivamente se asignaron los registros 39787 y 39788 de la colección del Herbario HUMO de la Universidad Autónoma del Estado de Morelos. En el sitio de colecta se cuantificó una población conformada por 63 individuos. Las colectas fueron determinadas mediante claves taxonómicas (Cubas, 1984) y corroboradas con descripciones precisas (Clements et al., 2001). Este material colectado, aunado a las referencias bibliográficas y a la observación realizada en el área de registro, permite reconocer que $U$. europaeus es un arbusto perenne que puede vivir hasta 20 años, su altura es hasta de $3 \mathrm{~m}$, su tallo grueso, verde, muy ramificado y armado con espinas primarias ramificadas en secundarias y terciarias de 1-4 cm de largo, rígidas y surcadas; raíz pivotante, gruesa, alcanza profundidades cercanas a los $50 \mathrm{~cm}$; hojas perennes aciculares (espinosas), generalmente más cortas que las espinas, las hojas se reducen a escamas o filoides en forma de espina; flores hermafroditas $1.5-2.5 \mathrm{~cm}$ de largo, solitarias, en racimos o panículas, creciendo en las espinas primarias, a veces en espinas secundarias, las flores con pedicelos pubescentes, con una bráctea basal a veces no perceptible y dos bractéolas de 2-6 × 2-7.5 mm, más pequeñas debajo del cáliz; cáliz 10-15 mm de largo, persistente, aplanado, amarillo con tricomas gruesos, el labio superior hasta $6 \mathrm{~mm}$ de ancho; corola 15-18 mm de largo, amarilla, compuesta por una quilla, dos alas laterales y un estandarte, alas más largas que el cáliz; androceo formado por 10 estambres que forman un tubo por donde pasa el estilo, el ovario desarrolla 12 óvulos; fruto una legumbre negra peluda, vainas oblongas $1-2 \mathrm{~cm}$ de largo, con pelos oscuros cuando madura, sostenidas por un cáliz persistente; las vainas con 1-7 semillas, de color marrón, brillantes, lisas, duras y resistentes al agua (Fig. 1).

El análisis realizado con el programa ArcGIS v. 10.2 (ESRI, 2013), para determinar el potencial de distribución geográfica o ubicación de otras poblaciones de esta especie en el estado de Morelos, permitió identificar 15 puntos con vegetación de Pinus-Quercus a una altitud de $2700 \mathrm{~m}$. Con los recorridos llevados a cabo en estos 15 puntos, se corroboró que, a la fecha, U. europaeus solo está presente en la localidad de Tres Marías, municipio Huitzilac (Fig. 2).

\section{Discusión}

Este estudio permite establecer que la especie invasora Ulex europaeus se suma, como una amenaza importante para la biodiversidad nacional, a la flora no nativa registrada en México. Los datos obtenidos en campo señalan que la floración y fructificación de U. europaeus se presenta en cualquier época del año, y en individuos juveniles de tan solo $15 \mathrm{~cm}$ con alto. Esta información es consistente con lo indicado por Tarayre et al. (2007). Estas características muestran su potencial reproductivo, su accesibilidad y disponibilidad de recursos y, en consecuencia, su alta capacidad competitiva (IPM, 2000).

Aun cuando a la fecha solo se encontró $U$. europaeus en la comunidad de Tres Marías en el municipio Huitzilac, es muy importante tomar en cuenta los antecedentes de distribución geográfica que esta especie tiene en otras partes del mundo. Al tratarse de una de las 100 especies invasoras más dañinas en el mundo, es fundamental mantener un monitoreo constante de esta especie en el estado de Morelos, tanto de los de los 63 individuos de la población colectada, como de los 15 escenarios geográficos similares a los de la localidad del registro. Esta especie se suma a la lista de especies invasoras presentes en el estado y en el país (March-Mifsut y Martínez-Jiménez, 2007; CONABIO, 2020; Ramírez-Rodríguez et al., 2020).

La referencia, en la base de datos del Instituto Forestal de las Islas del Pacífico, sobre la presencia de esta especie en México fue tomada del trabajo de Weber (2003). Las 21 citas bibliográficas en el trabajo de Weber (2003), sobre esta especie, corresponden a estudios realizados en Nueva Zelanda (9), Australia (6), Estados Unidos de Amé- 
rica (2) y el Océano Índico (1); las tres restantes versan sobre U. europaeus y su interacción con el herbicida Picloram (Acido 4-amino-3,5,6-tricloropicolínico) (Field y Hai, 1980), plantas invasoras (Cronk y Fuller, 1995) y malezas del mun- do (Holm et al., 1997). En ninguno de estos trabajos se hace referencia a la presencia de esta especie en México y a ningún ejemplar depositado en algún herbario. En la página Tropicos (Tropicos, 2020), la referencia de la pre-
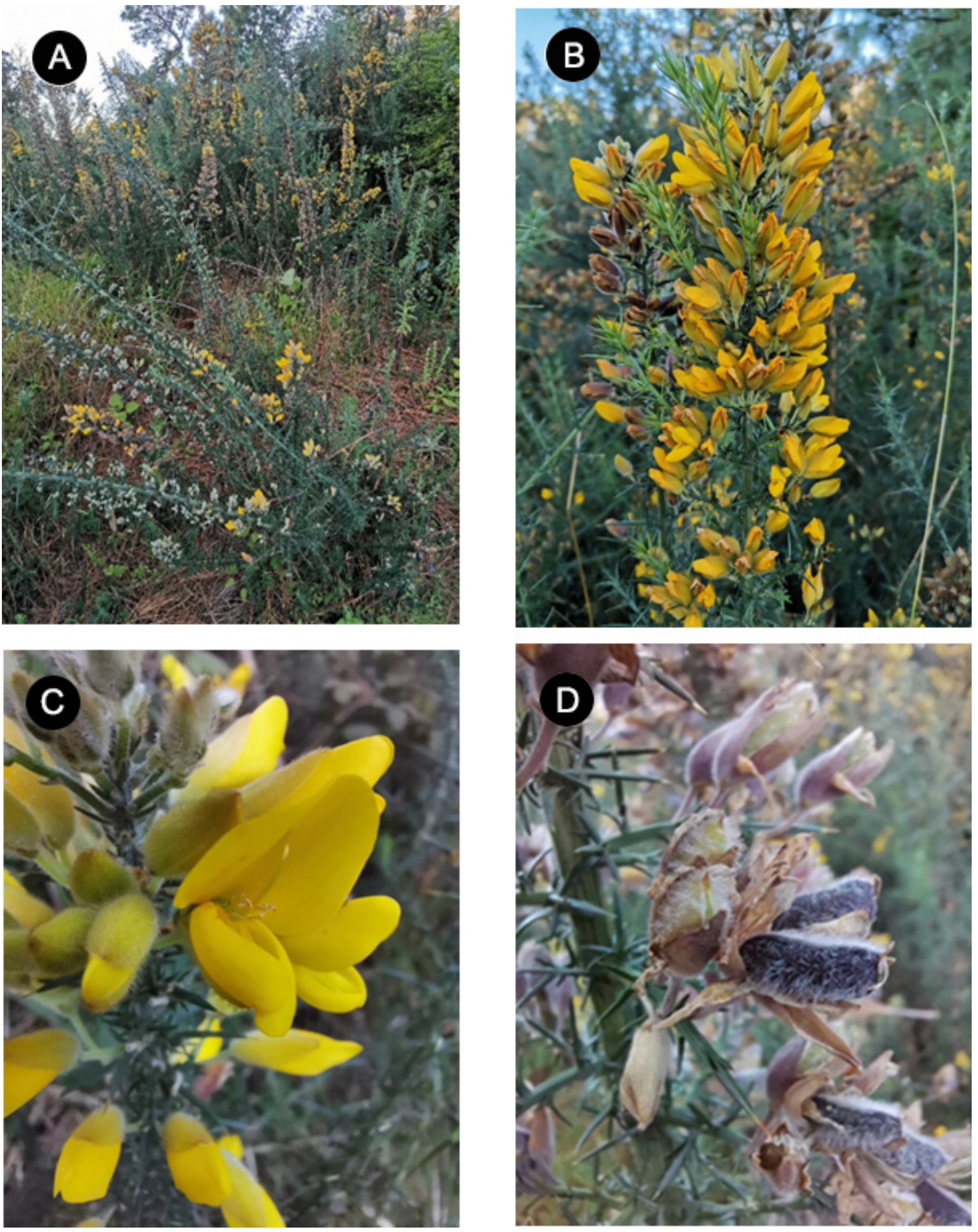

Figura 1: Ulex europaeus L.: A. entorno de la especie en la localidad de Tres Marías, municipio Huitzilac, Morelos, México; B. flores; C. detalle de la flor; D. fruto. 
$99^{\circ} 0^{\prime} 0^{\prime \prime W}$

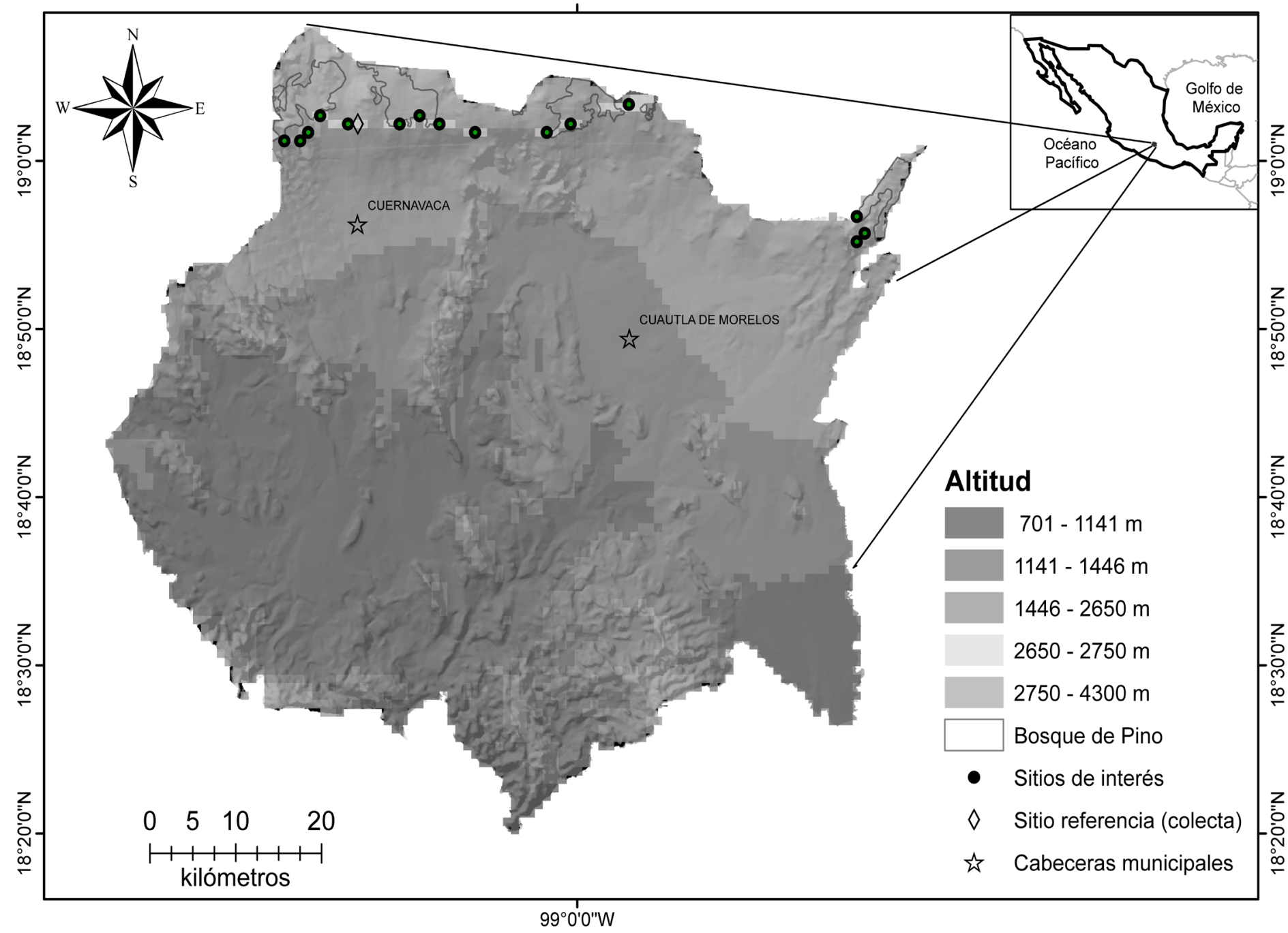

Figura 2: Localidad de registro de Ulex europaeus L. $(\diamond)$ en el estado de Morelos, México. Los 15 puntos $(\bullet)$ en este estado presentan condiciones geográfico-ambientales similares a las de la localidad de registro.

sencia de $U$. europaeus en México se basa en un trabajo de Fabaceae de Costa Rica (Zamora-Villalobos, 2010). Sin embargo, de la información presentada en esta base de datos se puede apreciar que, de los 40 registros mencionados, al menos 12 de ellos no cuentan con una colección botánica que acompañe a dicha referencia. Por tanto, no hay certeza de la presencia de esta especie en estas 12 localidades, entre las que se encuentra México. En este mismo sentido, en el trabajo de Atlan y Udo (2019) se menciona que en México U. europaeus es considerada como una especie invasora, como plaga agrícola, con impacto económico, con uso medicinal; sin embargo, en particular para México, no se ha encontrado ninguna publicación al respecto. Por otro lado, Sánchez-Blanco et al. (2012) en su estudio sobre las especies de leguminosas introducidas a México, con potencial para ser invasoras, no mencionan la presencia de U. europaeus en este país, condición que coincide con este estudio, ya que ni en la revisión de artículos académicos, ni en bases de datos, ni en páginas virtuales, ni en las revisiones físicas de las colecciones de los herbarios de la Universidad Nacional Autónoma de México (MEXU), de la Universidad Autónoma Metropolitana Iztapalapa (UAMIZ), de la Escuela Nacional de Ciencias Biológicas (ENCB) y de la Universidad Autónoma del Estado de Morelos HUMO, se encontraron ejemplares de $U$. europaeus colectados en México. 
Las posibles vías de introducción de U. europaeus a México pueden ser diversas. Entre ellas se puede mencionar la escasa vigilancia de los sistemas fronterizos y la falta de lineamientos para prevenir y controlar la entrada de especies al territorio como ocurre en varios países (Pérez-Fernández, 2012). Asimismo, en un mundo globalizado las vías de comunicación y transporte (terrestre, marítimo y aéreo), así como el turismo, favorecen la entrada de especies no nativas, ya sea de forma accidental o intencional (Torres-Rodríguez, 2009). En la dispersión de U. europaeus, las aves juegan un papel importante al trasladar sus semillas a grandes distancias (Parsons, 1958 citado por Hoshovsky, 1989). Además, las prácticas agrícolas, las actividades antropogénicas así como los afluentes de los ríos, facilitan el movimiento sin control de sus propágulos (Scott, 2005).

Las referencias acerca de efectos que genera esta especie sobre la dinámica de los ecosistemas (Atlan et al., 2015), los costos económicos que se han invertido para controlarla (Clements et al., 2001), la deforestación (Delgado-Ramírez, 2012) y el cambio climático (Mantyka-Pringle et al., 2015), indican que el futuro para la biodiversidad no es prometedor. Por ello, se destaca la importancia del registro de Ulex europaeus en México, y se pondera la necesidad de dar un seguimiento detallado a la población registrada de esta especie. La inversión económica que ahora se haga para controlar a esta especie será mínima en comparación con los gastos que se generarán en caso de no considerar los antecedentes en términos del daño ecológico, económico y humano que ya se ha registrado en otras naciones. Por ejemplo, en Chile se estima que, a causa de esta especie, cada año hay una pérdida anual mínima de cuatro millones de dólares (Cerda et al., 2017). De ahí la importancia de atender lo antes posible el problema que esta especie generará en México.

\section{Contribución de autores}

EMM y FLS realizaron la colecta y monitoreo de la especie en campo; MFT realizó los análisis de ArcGIS; ACG y ETS realizaron trabajo de campo y colecta de material vegetal para futuros estudios de variabilidad genética y filogeografía, respetivamente, y JRRR registró la primera colecta de la especie, coordinó el estudio en campo y elaboró el docu- mento base para la publicación. Todos los autores revisaron y enriquecieron el documento que se presenta.

\section{Financiamiento}

Esta investigación fue apoyada por fondos propios de los autores del estudio.

\section{Agradecimientos}

Los autores agradecen a la Universidad Autónoma del Estado de Morelos (UAEM) su compromiso con la protección de la biodiversidad. Se externa nuestro agradecimiento a Alejandro Flores Morales y Gabriel Flores Franco, por al apoyo académico brindado al primer autor.

\section{Literatura citada}

Altamirano, A., J. P. Cely, A. Etter, A. Miranda, A. Fuentes-Ramírez, P. Acevedo. C. Salas y R. Vargas. 2016. The invasive species Ulex europaeus (Fabaceae) shows high dynamism in a fragmented landscape of south-central Chile. Environmental Monitoring and Assessment 188: 495. DOI: https://doi. org/10.1007/s10661-016-5498-6

Araos, A., C. Cerda, O. Skewes, G. Cruz, P. Tapia y F. Baeriswyl. 2020. Estimated economic impacts of seven invasive alien species in Chile. Human Dimensions of Wildlife 25(4): 398403. DOI: https://doi.org/10.1080/10871209.2020.1740837

Atlan, A., N. Udo, B. Hornoy y C. Darrot. 2015. Evolution of the uses of gorse in native and invaded regions: what are the impact on its dynamics and management? Revue d'Ecologie (Terre et Vie) 70(suppl. 12): 1-16.

Atlan, A. y N. Udo. 2019. The invasive niche, a multidisciplinary concept illustrated by Gorse (Ulex europaeus). Diversity 11(9): 162. DOI: https://doi.org/10.3390/d11090162

Baeza, M. J., M. De Luis, J. Raventós y A. Escarré. 2002. Factors influencing fire behavior in shrublands of different stand ages and the implications for using prescribed burning to reduce wildfire risk. Journal of Environmental Management 65(2): 199-208. DOI: https://doi. org/10.1006/jema.2002.0545

Beckdorf, F. 1985. Aspectos biológicos de Ulex europaeus L. y de su antagonista Apio nulicis Forst. Tesis de licenciatura. Facultad de Ciencias Agrarias, Universidad Austral de Chile. Valdivia, Chile. 95 pp. 
Bowman, G., M. Tarayre y A. Atlan. 2008. How is the invasive gorse Ulex europaeus pollinated during winter? A lesson from its native range. Plant Ecology 197(2): 197-206. DOI: https://doi.org/10.1007/s11258-007-9370-1

Bridson, D. y L. Forman. 1998. The herbarium handbook. 3th ed., Royal Botanic Gardens. Kew, UK. 319 pp.

Broadfield, N. y M. T. McHenry. 2019. A world of gorse: persistence of Ulex europaeus in managed landscapes. Plants 8(11): 523. DOI: https://doi.org/10.3390/plants8110523

Camelo-Salamanca, D. 2015. Evaluación del estado sucesional actual de las áreas restauradas y con invasión previa de Ulex europaeus L. en los Cerros Orientales de Bogotá. Tesis de maestría. Pontificia Universidad Javeriana. Bogotá, Colombia. 148 pp.

Cerda, C., G. Cruz, O. Skewes, A. Araos, P. Tapia, F. Baeriswyl y P. Critician. 2017. Especies exóticas invasoras en Chile como un problema económico: valoración preliminar de impactos. Revista del Jardín Botánico Chagual 15: 12-22.

Clements, D. R., D. J. Peterson y R. Prasad. 2001. The biology of Canadian weeds. 112. Ulex europaeus L. Canadian Journal of Plant Science 81(2): 325-337. DOI: https://doi.org/10.4141/ P99-128

CONABIO. 2020. Especies exóticas invasoras ¿Cuáles son? Comisión Nacional para el Conocimiento y Uso de la Biodiversidad (CONABIO). Cd. Mx., México. https://www. biodiversidad.gob.mx/especies/Invasoras/cuales-son (consultado agosto de 2021).

Cronk, Q. C. B. y J. L. Fuller. 1995. Plant invaders. Chapman \& Hall. London, UK. 241 pp.

Cruz-Cárdenas, G., J. L. Villaseñor, L. López-Mata, E. MartínezMeyer y E. Ortiz. 2014. Selección de predictores ambientales para el modelado de la distribución de especies en Maxent. Revista Chapingo, Serie Ciencias Forestales y del Ambiente 20(2): 187-201. DOI: https://doi.org/10.5154/r. rchscfa.2013.09.034

Cubas, D. P. 1984. Estudio taxonómico de los géneros Ulex L. Y Stauracanhus Link. en la Península Ibérica. Tesis doctoral. Facultad de Ciencias Biológicas, Universidad Complutense de Madrid. Madrid, España. 287 pp.

Cubas, P., C. Pardo y H. Tahiri. 2005. Genetic variation and relationships among Ulex (Fabaceae) species in southern Spain and northern Morocco assessed by chloroplast microsatellite (cPSSR) markers. American Journal of Botany 92(12): 2031-2043. DOI: https://doi.org/10.3732/ ajb.92.12.2031

Delgado-Ramírez, J. P. 2012. Deforestación y fragmentación de selvas en el sur de la Península de Yucatán, México (1990-2006). Tesis doctoral. El Colegio de la Frontera Sur. Chetumal, Quintana Roo, México. 53 pp.

Díaz-Espinoza, A. M. 2009. Rasgos de historia de vida y ecología de las invasiones de Ulex europaeus L. In: Vargas, O. O., A. León y A. Díaz-Espinosa (eds.). Restauración ecológica en zonas invadidas por retamo espinoso y plantaciones forestales de especies exóticas. Grupo de Restauración Ecológica, Universidad Nacional de Colombia. Bogotá, Colombia. Pp. 59-67.

Ellner, S. y A. Sasaki. 1996. Patterns of genetic polymorphism maintained by fluctuating selection with overlapping generations. Theoretical Population Biology 50(1): 31-65. DOI: https://doi.org/10.1006/tpbi.1996.0022

ENCB. 2020. Herbario de la Escuela Nacional de Ciencias Biológicas. Instituto Politécnico Nacional. Cd. Mx., México. http://www. herbario.encb.ipn.mx/ (consultado abril de 2020).

ESRI. 2013. ArcGIS ver. 10.2 Environmental Systems Research Institute. Redlands, USA.

Fernández Prieto, J. A., M. L. Vera, M. J. Álvarez Martínez, T. E. Díaz, M. A. Fernández-Casado, M. C. Fernández-Carvajal, M. I. Gutierrez Villarias y H. S. Nava. 1993. Chromosome numbers and geographical distribution of Ulex europaeus subsp. europaeus. Botanical Journal of the Linnean Society 113(1): 35-39. DOI: https://doi.org/10.1111/j.1095-8339.1993. tb00327.x

Field, R. J. y P. H. Hai. 1980. The preferential accumulation of picloram at sites of active growth in gorse (Ulex europaeus L.). Weed Research 20(3): 177-182. DOI: https://doi. org/10.1111/j.1365-3180.1980.tb00065.x

Gómez, J., R. Minhorst y I. P. Iglesias. 2009. Evaluación de diferentes sistemas de elaboración de sustratos de cultivo obtenidos a partir de tojo (Ulex europaeus L.). Spanish Journal of Rural Development 1: 188-204.

González-Hernández, M. P., R. Romero, M. Rodríguez-Guitián y A. Rigueiro. 2004. Medicinal use of some plants in Galicia (NW Spain). Acta Horticulturae 629: 63-75. DOI: https://doi. org/10.17660/ActaHortic.2004.629.8 
Haldane, J. B. S. y S. D. Jayakar. 1963. Polymorphism due to selection of varying direction. Journal of Genetics 58(2): 237-242. DOI: https://doi.org/10.1007/BF02986143

Hendry, A. P. y T. Day. 2005. Population structure attributable to reproductive time: isolation by time and adaptation by time. Molecular Ecology 14(4): 901-916. DOI: https://doi. org/10.1111/j.1365-294X.2005.02480.x

Herrera, J. 1999. Fecundity above the species level: ovule number and brood size in the Genisteae (Fabaceae: Papilionoideae). International Journal of Plant Sciences 160(5): 887-896. DOI: https://doi.org/10.1086/314183

Hill, R. L., A. H. Gourlay y L. Martin. 1991. Seasonal and geographic variation in the predation of gorse seed, Ulex europaeus L., by seed weevil Apio nulicis Forst. New Zealand Journal of Zoology 18(1): 37-43. DOI: https://doi.org/10.1080/03014 223.1991.10757946

Hill, R. L., A. H. Gourlay y R. J. Barker. 2001. Survival of Ulex europaeus seeds in the soil at three sites in New Zealand. New Zealand Journal of Botany 39(2): 235-244. DOI: https:// doi.org/10.1080/0028825X.2001.9512734

Hill, R. L., J. Ireson, A. W. Sheppard, A. H. Gourlay, H. Norambuena, G. P. Markin, R. Kwong y E. M. Coombs. 2008. A global view of the future for biological control of gorse, Ulex europaeus L. In: Proceedings of the XII international symposium on biological control of weeds. CAB International. Wallingford, UK. Pp. 680-686.

Holm, L. G., J. Doll, E. Holm, J. Pancho y J. Herberger. 1997. World Weeds. John Wiley \& Sons. New York, USA. 1152 pp.

Hoshovsky, M. 1989. Element Stewardship Abstract (ESA) for Ulex europaeus: Gorse. The Nature Conservancy. Arlington, USA. $22 \mathrm{pp}$.

IPM. 2000. Integrated Pest Management Information Service. Noxious Weed Integrated Vegetation Management (IVM) Guide: Gorse. https://www2.gov.bc.ca/gov/content/ environment/pesticides-pest-management/businessindustry/sector-specific-tools-guides (consultado marzo de 2019).

IUCNGISD. 2020. Global Invasive Species Database (IUCNGISD). Invasive Species Specialist Group. http://www.iucngisd.org/ gisd/ (consultado marzo de 2019).

Johnston, F. H., S. B. Henderson, Y. Chen, J. T. Randerson, M. Marlier, R. S. DeFries, P. Kinney, D. M. J. S. Bowman y M. Brauer.
2012. Estimated global mortality attributable to smoke from landscape fires. Environmental Health Perspectives 120(5): 695-701. DOI: https://doi.org/10.1289/ehp.1104422

K. 2021. Herbarium. Royal Botanic Gardens. Kew, UK. https:// www.kew.org/science/collections-and-resources/ collections/herbarium (consultado abril 2021).

LE. 2021. Vascular Plants Herbarium. Komarov Botanical Institute of the Russian Academy of Sciences. Moscú, Rusia. http:// www.binran.ru (consultado enero de 2021).

Leung, B., D. M. Lodge, D. Finnoff, J. F. Shogren, M. A. Lewis y G. Lamberti. 2002. An ounce of prevention or a pound of cure: bioeconomic risk analysis of invasive species. Proceedings of the Royal Society of London, Series B: 269(1508): 24072413. DOI: http://doi.org/10.1098/rspb.2002.2179

López, N. y A. López. 2012. Uso de un sustrato alternativo a la turba para la producción viverística de plantas hortícolas y aromáticas. Recursos Rurales 8: 31-37.

Lot, A. y F. Chiang. 1986. Manual de Herbario: Administración y manejo de colecciones, técnicas de recolección y preparación de ejemplares botánicos. Consejo Nacional de la Flora de México, A.C. México, D.F. México. 142 pp.

Lowe, S., M. Browne, S. Boudjelas y M. De Poorter. 2000. 100 of the world's worst invasive alien species, a selection from the global invasive species database. Invasive Species Specialist Group (ISSG) a specialist group of the Species Survival Commission (SSC) of the World Conservation Union (IUCN). Auckland, New Zealand. Pp. 1-11. http://www.issg. org/pdf/publications/worst_100/english_100_worst.pdf (consultado mayo de 2020).

LOWO. 2020. Legumes of the world online. Royal Botanic Gardens, Kew. Kew, UK. http://plantsoftheworldonline.org/taxon/ urn:Isid:ipni.org:names:23746-1 (consultado abril de 2020).

Madrigal, J., E. Marino, M. Guijarro, C. Hernando y C. Díez. 2012. Evaluation of the flammability of gorse (Ulex europaeus L.) managed by prescribed burning. Annals of Forest Science 69(3): 387-397. DOI: https://doi.org/10.1007/s13595-0110165-0

Mantyka-Pringle, C. S., P. Visconti, M. Di Marco, T. G. Martin, C. Rondinini y J. R. Rhodes. 2015. Climate change modifies risk of global biodiversity loss due to land-cover change. Biological Conservation 187: 103-111. DOI: https://doi. org/10.1016/j.biocon.2015.04.016 
March-Mifsut, I. y M. Martínez-Jiménez. 2007. Especies invasoras de alto impacto a la biodiversidad: prioridades en México. Instituto Mexicano de Tecnología del Agua. Jiutepec, México. http://repositorio.imta.mx/handle/20.500.12013/1619 (consultado noviembre de 2019).

Maxwell, H. 1995. Ulex. In: Cullen, J., J. C. M. Alexander, A. Brady, C. D. Brickell, P. S. Green, V. H. Heywood y P. F. Yeo (eds.). The European Garden Flora. Cambridge University Press. Cambridge, UK. Pp. 550.

MEXU. 2020. Herbario Nacional de México. Instituto de Biología, Universidad Nacional Autónoma de México. Cd. Mx., México. http://www.ib.unam.mx/botanica/herbario/ (consultado septiembre de 2020).

Misset, M. T. y J. P. Gourret. 1996. Flow cytometric analysis of the different ploidy levels observed in the genus Ulex L. Faboideae-Genisteae in Brittany (France). Plant Biology 109(1): 72-79. DOI: https://doi. org/10.1111/j.1438-8677.1996.tb00872.x

MHNH. 2021. Museo Natural de Historia Natural (MHNH). Santiago, Chile. https://www.mnhn.gob.cl/colecciones (consultado septiembre de 2020).

MO. 2020. Missouri Botanical Garden Herbarium (MO). Missouri Botanical Garden. San Louis, USA. https://www. missouribotanicalgarden.org/plant-science/plant-science/ resources/herbarium.aspx (consultado junio de 2020).

Naturalista. 2020. Naturalista. Comisión Nacional para el Conocimiento y Uso de la Biodiversidad (CONABIO). Cd. Mx., México. http://www.naturalista.mx (consultado julio de 2020).

Niño, Y., L. T. Vega y B. Ruiz. 2018. Evaluation of the energy potential of the gorse (Ulex europaeus) in the generation of electrical energy by gasification. Chemical Engineering Transactions 64: 481-486. DOI: https://doi.org/10.3303/ CET1864081

Norambuena, H. y G. L. Piper. 2000. Impact of Apio nulicis Forster on Ulex europaeus L. seed dispersal. Biological Control 17(3): 267-271. DOI: https://doi.org/10.1006/bcon.1999.0801

NY. 2020. C.V. Starr Virtual Herbarium. New York Botanical Garden. Nueva York, EUA. http://sweetgum.nybg.org/science/vh/ (consultado agosto de 2020).

Osorio, M. y L. A. Cerda. 1984. Icerya purchasi M. y otros organismos asociados a Ulex europaeus L. y Cytisus monspessulanus L. Bosque 5(2): 110-114. DOI: https://doi. org/10.4206/bosque.1984.v5n2-06

P. 2020. The vascular plants collection (P) at the Herbarium of the Muséum National d'Histoire Naturelle. Paris, Francia. https://science.mnhn.fr/institution/mnhn/collection/p/ item/search/form (consultado mayo de 2020).

Pérez-Fernández, M. A. 2012. Control de especies invasoras. In: Camprodon, J., M. T. Ferreira-Isa y M. Ordeix-Cerm (eds.). Restauración y gestión ecológica fluvial. IsaPress. Lisboa, Portugal. Pp. 138-154.

Philip, A. y P. A. Thomas. 2018. Ulex europaeus. Institute of Pacific Island Forestry. http://www.hear.org/pier/species/ulex_ europaeus.htm (consultado julio de 2020).

Piñero, D., A. Barahona, L. E. Eguiarte, A. Rocha-Olivares y R. Salas-Lizana. 2008. La variabilidad genética de las especies: aspectos conceptuales y sus aplicaciones y perspectivas en México, en Capital Natural de México, Vol. I: Conocimiento actual de la biodiversidad. Comisión Nacional para el Conocimiento y Uso de la Biodiversidad (CONABIO). Cd. Mx., México. pp. 415-435.

Ramírez-Morán, N. A., M. León-Gómez y R. Lücking. 2016. Efecto del retamo espinoso (Ulex europaeus) sobre las aves de borde en un bosque alto andino. Ornitología Colombiana 10: $11-25$

Ramírez-Rodríguez, R., F. Ocampo-Bautista, B. M. Rojas-Flores, G. Flores-Franco, E. Tovar-Sánchez y A. D. Sánchez-Popoca. 2020. Flora arbórea no nativa, un potencial riesgo para la biodiversidad. La biodiversidad en Morelos. Estudio de Estado 2, Vol. III. Comisión Nacional para el Conocimiento y Uso de la Biodiversidad (CONABIO). Cd. Mx., México. Pp. 234-240.

Sánchez-Blanco, J., C. Sánchez-Blanco, M. Sousa S. y F. J. Espinosa-García. 2012. Assessing introduced Leguminosae in Mexico to identify potentially high-impact invasive species. Acta Botanica Mexicana 100: 41-77. DOI: https:// doi.org/10.21829/abm100.2012.31

Sandrey, R. 1985. Biological control of gorse: an ex-ante evaluation. Agricultural Economics Research Unit Research Report No. 172. Lincoln College. Canterbury, New Zealand. $96 \mathrm{pp}$.

Scott, B. 2005. The Temporal Effects of Ulex europaeus on soil properties, and Modeling Impact of Invasive Species with 
Respect to Time. MSc thesis. Universidad de Washington. Seattle, USA. $112 \mathrm{Pp}$.

SMN. 2019. Servicio Meteorológico Nacional. Comisión Nacional del Agua. Gobierno de México. https://smn.conagua.gob. mx/es/ (consultado noviembre de 2019).

Soto, B. y F. Diaz-Fierros. 1997. Soil water balance as affected by throughfall in gorse (Ulex europaeus, L.) shrubland after burning. Journal of Hydrology 195(1-4): 218-231. DOI: https://doi.org/10.1016/S0022-1694(96)03232-5

Springer, D. J. 1985. Human influence on the distribution of gorse (Ulex europaeus L.) along the Mendocino County Coast California. Thesis for degree of Master of Arts. Department of Geography, University of Montana. Montana, USA. 81 pp.

Suguru-Yonezawa, I. Maruyama, K. Sakae, A. Igata, P. W. Majerus y E. Sato. 1987. Thrombomodulin as a marker for vascular tumors: comparative study with factor VIII and Ulex europaeus I lectin. American Journal of Clinical Pathology 88(4): 405-411. DOI: https://doi.org/10.1093/ajcp/88.4.405

Tarayre, M., G. Bowman, A. Schermann-Legionnet, M. Barat y A. Atlan. 2007. Flowering phenology of Ulex europaeus: ecological consequences of variation within and among populations. Evolutionary Ecology 21(3): 395-409. DOI: https://doi.org/10.1007/s10682-006-9109-9

Thiers, B. 2021 (continuously updated). Index Herbariorum: A global directory of public herbaria and associated staff. New York Botanical Garden's Virtual Herbarium. New York, USA. http://sweetgum.nybg.org/ih/ (consultado noviembre de 2021).
Torres Rodríguez, N. A. 2009. Banco de semillas germinable en áreas invadidas por retamo espinoso (Ulex europaeus) con diferentes edades de quema (alrededores del embalse de Chisacá Bogotá-localidad de Usme). Tesis de licenciatura. Facultad de Ciencias, Universidad Militar Nueva Granada. Santa Fe de Bogotá, Colombia. 110 pp.

Tropicos. 2020. Tropicos.org. Missouri Botanical Garden. Missouri, USA. https://tropicos.org (consultado noviembre de 2020).

US. 2020. United States National Herbarium Smithsonian Institution. Washington, D.C., USA. https://naturalhistory. si.edu/research/botany (consultado mayo de 2020).

Villaseñor, J. L. y E. Ortiz. 2014. Biodiversidad de las plantas con flores (División Magnoliophyta) en México. Revista Mexicana de Biodiversidad 85: 134-142. DOI: http://dx.doi. org/10.7550/rmb.31987 (consultado enero de 2020).

Weber, E. 2003. Invasive plants of the World. CABI Publishing International. Wallingford, UK. 548 pp.

Wolf, C. 1988. Empleo de Espino Amarillo (Ulex europaeus) en alimentación de bovinos: Efectos tóxicos y consumo. Tesis de licenciatura. Facultad de Ciencias Veterinarias, Universidad Austral de Chile. Valdivia, Chile. 24 pp.

Zamora-Villalobos, N. 2010. Fabaceae. In: Hammel, B. E., M. H. Grayum, C. Herrera y N. Zamora Villalobos (eds.). Manual de plantas de Costa Rica. Monographs in systematic botany from the Missouri Botanical Garden 119(5): 395-775. 